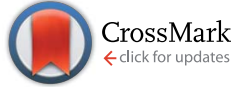

Cite this: Chem. Sci., 2016, 7, 1309

Received 23rd September 2015 Accepted 9th November 2015

DOI: $10.1039 / \mathrm{c} 5 \mathrm{sc} 03604 \mathrm{f}$

www.rsc.org/chemicalscience

\section{Fusing N-heteroacene analogues into one "kinked" molecule with slipped two-dimensional ladder-like packing $\uparrow$}

\author{
Jing Zhang, $\$^{\mathrm{a}}$ Chengyuan Wang, $\ddagger^{\mathrm{a}}$ Guankui Long, ${ }^{\mathrm{a}}$ Naoki Aratani, ${ }^{\mathrm{b}}$ Hiroko Yamada ${ }^{\mathrm{b}}$ \\ and Qichun Zhang*ac
}

\begin{abstract}
An unexpected "kinked" N-heteroacene with a slipped two-dimensional ladder-like packing feature is produced from the conventional condensation reaction. The as-obtained compound $\left[2,2^{\prime}\right] \mathrm{bi}(5,12-$ bis(TIPS)piperazin-3-one[2,3-b]phenazine) (2BPP) consists of two identical backbones (5,12-bis(TIPS) piperazin-3-one[2,3-b]phenazine), which are fused together through a $\mathrm{C}=\mathrm{C}$ double bond and two intramolecular $\mathrm{H}$-bonds. A study on the charge carrier transport indicates that a 2BPP single crystal has a hole mobility up to $0.3 \mathrm{~cm}^{2} \mathrm{~V}^{-1} \mathrm{~s}^{-1}$, while theoretical calculations suggest that this compound might possess potential well-balanced ambipolar charge-transport characteristics.
\end{abstract}

In past decades, polycyclic aromatic hydrocarbons (PAHs) and $\mathrm{N}$-heteroacene derivatives have attracted a lot of attention, being widely investigated for applications in organic field-effect transistors (OFETs), organic resistance memories (ORMs), organic light-emitting diodes (OLEDs) and organic photovoltaics (OPVs). ${ }^{1}$ Pentacene and its soluble analogue 6,13-bis(triisopropylsilylethynyl)pentacene (TIPS-pentacene) are two of the most popular small-molecule organic semiconductors, exhibiting superior hole transporting mobilities $\left(>1 \mathrm{~cm}^{2} \mathrm{~V}^{-1} \mathrm{~s}^{-1}\right)$ due to their close intermolecular packing, which is supposed to favor charge carrier transport. ${ }^{2}$ Thus, tailoring one molecular structure to achieve a larger $\pi$-conjugated system or a more condense packing mode is highly desirable. Longer acenes are presumed to possess enhanced intermolecular $\pi-\pi$ overlap in the solid state, which could lead to high charge carrier mobilities. ${ }^{3}$ However, the instability of large acenes strongly hampers their application in organic electronics. To address this issue, two-dimensional (2D) acenes with both more sextets and sufficient stability are proposed. 2D acenes and their derivatives have large, planar $\pi$ surfaces, which can provide the increased intermolecular surface overlapping and effectively increase electron delocalization, potentially resulting in

${ }^{a}$ School of Materials Science and Engineering, Nanyang Technological University, Singapore, 639798, Singapore.E-mail: qczhang@ntu.edu.sg

${ }^{b}$ Graduate School of Materials Science, Nara Institute of Science and Technology, Ikoma, 630-0192, Japan

${ }^{c}$ Division of Chemistry and Biological Chemistry, School of Physical and Mathematical Sciences, Nanyang Technological University, Singapore, 637371, Singapore

$\dagger$ Electronic supplementary information (ESI) available: Details of experimental procedure, high-resolution mass spectrometry, ${ }^{1} \mathrm{H} \quad \mathrm{NMR},{ }^{13} \mathrm{C} \quad \mathrm{NMR}$, computational methodology. CCDC 1420681. For ESI and crystallographic data in CIF or other electronic format see DOI: 10.1039/c5sc03604f

\$ These authors contributed equally to this work. enhanced charge carrier transporting properties. For example, single-crystal OFETs of a bistetracene derivative reveal a higher hole mobility up to $6.1 \mathrm{~cm}^{2} \mathrm{~V}^{-1} \mathrm{~s}^{-1}$ with a remarkable $I_{\mathrm{on}} / I_{\text {off }}$ ratio of $10^{7} .{ }^{4} \mathrm{~N}$-heteroacenes have also been investigated and show p-type, ambipolar or n-type OFET behaviours as the number and position of $\mathrm{N}$ atoms in the backbone varies. ${ }^{5}$ OFETs based on a vacuum-deposited $N$-substituted TIPS-pentacene analogue show electron mobilities in the range of $1.0-3.3 \mathrm{~cm}^{2} \mathrm{~V}^{-1} \mathrm{~s}^{-1}$ under vacuum and $0.3-0.5 \mathrm{~cm}^{2} \mathrm{~V}^{-1} \mathrm{~s}^{-1}$ in ambient air. To date, most of the explored $\mathrm{N}$-heteroacenes are linearly-fused systems, which can be prepared through the condensation reaction between ortho-diamine based acenes and ortho-diketone, ortho-dihydroxy, ortho-dicyano, or ortho-dihalogen substituted acenes (Scheme 1). ${ }^{6}$ However, in such synthetic conditions, $\mathrm{N}$-heteroacenes with unusual shapes are rarely discovered, not to mention studied for their applications. In this report, we present an unexpected "kinked" N-heteroacene with a ladderlike packing feature, which was prepared from the conventional condensation reaction. Its new zigzag structure, physical properties and charge transport capabilities have been carefully investigated.

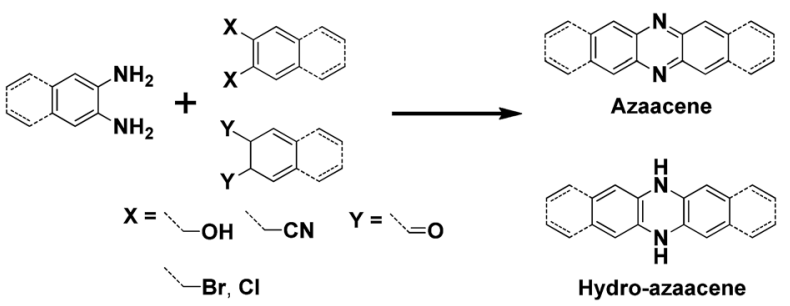

Scheme 1 Representative synthetic route to linearly fused $\mathrm{N}$ heteroacenes. 
In our previous study, we have already reported that linearlyfused N-heteroacene THNQ (shown in Scheme 2) can be prepared through the condensation reaction between 1,4-bis(TIPS)-2,3-diaminophenazine (BTDP) and hexaketocyclohexane (HKCH), in which we believe that the steric effect of the TIPS group plays a crucial role. ${ }^{7}$ Continuing in this direction, we further studied the reactions between the smaller 1,4-bis(TIPS)-acene-2,3-diamines and HKCH. ${ }^{8}$ Surprisingly, when the short 1,4-bis(TIPS)-diaminonaphthalene was used as the starting material, both the star-shaped compound (all the carbonyl groups substituted by $\mathrm{N}$ atoms in a yield of $26 \%$ ) and the linearly-fused product (yield of $17 \%$ ) were obtained. These results suggest that there might have been other factors besides the steric effect, which could also affect the condensation reaction between BTDP and HKCH. In this situation, we might miss some important unknown N-heteroacene products. Thus, we reinvestigated this type of reaction and discovered a meaningfully "kinked" compound [2,2']bi(5,12-bis(TIPS)piperazin-3one[2,3-b]phenazine) (2BPP). To the best of our knowledge, this is the first report of the generation of a meaningfully "kinked" $\mathrm{N}$-heteroacene through the conventional substitution reaction.

As shown in Scheme 2, the formation of 2BPP might undergo an unstable intermediate (UI) step, in which the diketone could be eliminated instead of participating in the further condensation reaction with the amine groups. The as-formed intermediate could be converted into the final product 2BPP through a rotation and radical pathway (initiated by light). The possible mechanism has been provided in the ESI (Scheme S1†). Note that the proposed mechanism is different from the previously reported mechanism to eliminate the diketone. ${ }^{9}$ The novel 2BPP was obtained in a low yield of $2.3 \%$ and was fully characterized by high-resolution mass (HR-MS) spectrometry, ${ }^{1} \mathrm{H}$ NMR and ${ }^{13} \mathrm{C}$ NMR spectroscopy, and single-crystal analysis. It is noteworthy that two 5,12-bis(TIPS)piperazin-3-one[2,3- $b]$ phenazine moieties are linked together by one double bond, which makes 2BPP fully $\pi$-conjugated along the backbone. In addition, the expected $\mathrm{H}$-bonds along both sides were assumed to stabilize the large heteroacene system.

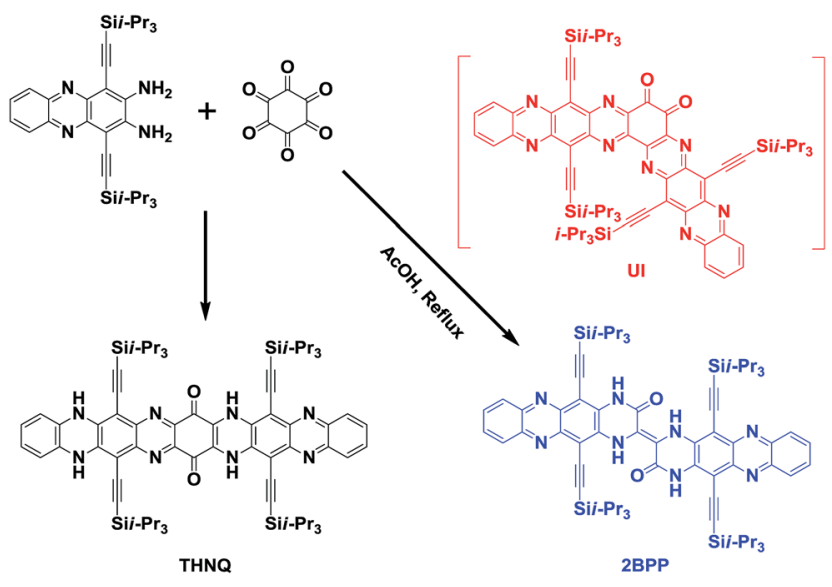

Scheme 2 Synthetic route to 2BPP.
Single crystals (CCDC: $1420681 \dagger$ ) of 2BPP suitable for singlecrystal X-ray diffraction analysis were obtained by diffusing the poor solvent acetonitrile into a toluene solution. 2BPP crystallizes in a triclinic unit cell, space group $P \overline{1}(2)$. The molecular structure of 2BPP is shown in Fig. 1a. The length of the bond between the $\mathrm{C} 2$ and $\mathrm{C} 2$ ' atoms is $1.35 \AA$, which is identical to the length of a common $\mathrm{C}=\mathrm{C}$ bond $(1.35 \AA)$, suggesting that the two (5,12-bis(TIPS)piperazin-3-one[2,3- $b]$ phenazine, BPP) moieties are connected by a $\mathrm{C} 2=\mathrm{C} 2{ }^{\prime}$ bond. The distance between $\mathrm{H} 1$ and $\mathrm{O} 1$ or $\mathrm{H}^{\prime}$ and $\mathrm{O}^{\prime}$ is $1.95 \AA$, indicating the existence of an intramolecular H-bond. Clearly, the formation of the intramolecular H-bonds with a six-member ring configuration is helpful for stabilizing the planar molecular shape and supports the electron delocalization. In fact, the as-prepared molecule possesses a good planarity from the side view (Fig. 1b). Only a slight twist of 2BPP can be observed, and the dihedral angle between the BPP moiety and the $\mathrm{C} 2=\mathrm{C} 2$ ' bond is $\sim 2.42^{\circ}$. As shown in Fig. 1c, 2BPP exhibits a slipped 2-D $\pi$-stacking motif, similar to that observed for some soluble TIPS-pentacene derivatives. The interplanar distance in 2BPP is $\sim 3.27 \AA$ along the $b$ axis, less than that of the typical distance for van der Waals interactions, while the centre-to-centre distance between two adjacent molecules is $\sim 14.65 \AA$. The slipping angle of two adjacent $\pi$-conjugated BPPs is $\sim 45^{\circ}$, contributing to a significant molecular overlap and ensuring the strong $\pi-\pi$ interaction. While viewing along the $a$ axis, the distance between two adjacent molecules is $\sim 3.33 \AA$ and in this stacking mode, the

a)

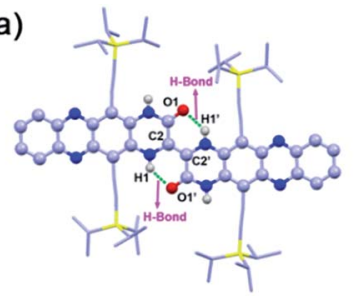

b)

c)

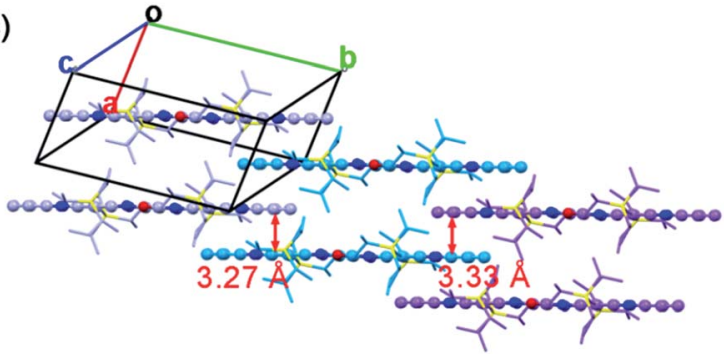

d)

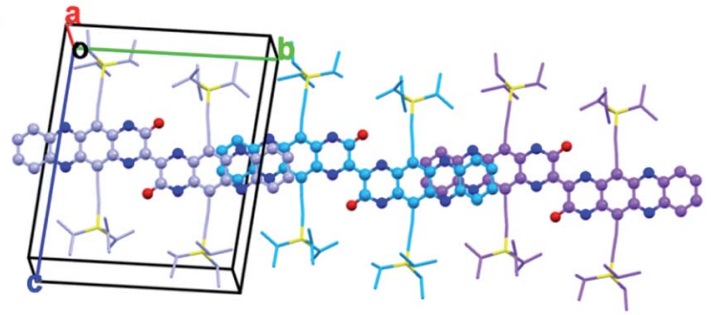

Fig. 1 (a) Top \& (b) side views of the 2BPP molecular structure (green dash lines represent the intramolecular $\mathrm{H}$-bond). (c) Interlayer distances between the neighbouring molecules. (d) View of molecular stacking along the $a$ axis. 
characterized centre-to-centre distance is about $17.61 \AA$ with a much smaller slipping angle $\left(\sim 30^{\circ}\right)$ between two interactional individual BPPs leading to poorer electronic coupling. 2BPP molecules interact with each other to form two-layer BPP units. The face-to-face stacking mode results in overlapping between the second layer units and the first layer BPPs (Fig. 1d).

Fig. 2a is the UV-vis absorption spectrum of $2 \mathbf{B P P}$ in $\mathrm{CH}_{2} \mathrm{Cl}_{2}$. 2BPP has two maximum absorption bands with the maximum absorption at $627 \mathrm{~nm}$ and $685 \mathrm{~nm}$. The onset of absorption is $725 \mathrm{~nm}$, from which the optical bandgap $\left(E_{\mathrm{g}}\right)$ can be determined to be $1.71 \mathrm{eV}$. The strong absorption from $650 \mathrm{~nm}$ to $725 \mathrm{~nm}$ is similar to that of other hydro-azaacenes, and probably comes from the intramolecular charge transfer. The electrochemical properties of 2BPP were investigated by cyclic voltammetry (CV, Fig. 2b). Unlike other hydro-azaacenes, 2BPP shows no obvious oxidative peaks. One irreversible reductive peak can be observed with an onset potential $\left(v s . \mathrm{Fc}^{+} / \mathrm{Fc}\right)$ of $-1.29 \mathrm{~V}$, which determines the LUMO level of $2 \mathbf{B P P}$ to be $-3.51 \mathrm{eV}$. The HOMO level was calculated to be $-5.22 \mathrm{eV}$ from the LUMO level and $E_{\mathrm{g}}$. The geometry structure of 2BPP was optimized by DFT calculations (B3LYP/6-31G*), ${ }^{10}$ and a following frequency analysis was performed to ensure that the optimized structures were the stable states. As shown in Fig. S3, † the LUMO coefficient delocalizes on the whole conjugated backbone, while the HOMO coefficient mainly distributes on the middle electron-rich area. Table 1 summarizes the experimental and theoretical calculated energy levels of 2BPP. In both the experimental and theoretical calculated results, 2BPP has relatively high HOMO levels $(-5.22 \mathrm{eV}$ for the experimental, and $-5.02 \mathrm{eV}$ for the calculated result) and a moderate band gap, which indicates 2BPP could be used as a promising suitable semiconducting material.

Crystalline ribbons of 2BPP were grown on an octadecyltrichlorosilane (OTS)-treated $\mathrm{SiO}_{2} / \mathrm{Si}$ substrate by a drop-casting method. OTS was used to form a siloxane self-assembled monolayer (SAM) on the $\mathrm{SiO}_{2}$ layer, which could promote and facilitate molecular self-organization during the growth of ribbons. The optical and AFM images in Fig. 3a and b show that the 2BPP ribbons display a rhombic shape with unambiguous boundaries, indicating the high quality of the ribbons. The asobtained micro/nanoribbons are several to tens of micrometers in width, and several tens to hundreds of micrometers in length. Three sharp and strong diffraction peaks at $2 \theta=5.19$, 10.32 , and 15.45 degrees are observed in the out of plane X-ray diffraction patterns (XRD) (Fig. 3c), which could be assigned as
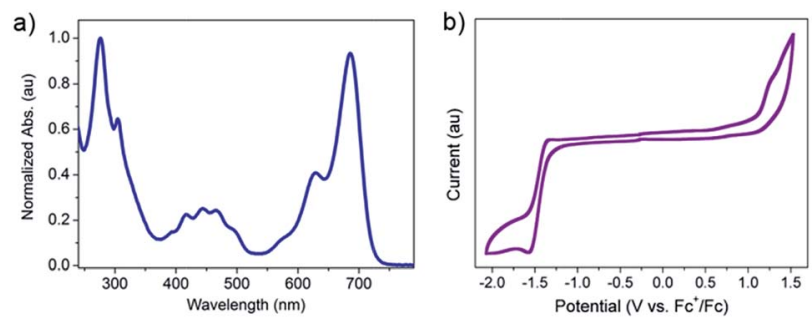

Fig. 2 (a) Normalized UV-vis absorption spectrum of 2BPP in $\mathrm{CH}_{2} \mathrm{Cl}_{2}$. (b) Cyclic voltammetric (CV) curve of 2BPP in anhydrous $\mathrm{CH}_{2} \mathrm{Cl}_{2}$.
Table 1 Summarized energy levels of 2BPP

\begin{tabular}{llll}
\hline Value & HOMO $(\mathrm{eV})$ & LUMO $(\mathrm{eV})$ & $E_{\mathrm{g}}(\mathrm{eV})$ \\
\hline Experimental & -5.22 & -3.51 & 1.71 \\
Calculated & -5.02 & -2.99 & 2.04
\end{tabular}

the (001), (002) and (003) planes, respectively, according to the information from the single-crystal structure. All three peaks in the pattern are well indexed along the (001) lattice plane, indicating that the ribbon-like crystals have high crystallinity. The spacing distance of the (001) plane is $17.13 \AA$, which is in agreement with the lattice parameters of the $c$-axis $(17.27 \AA)$, meaning that the molecules stand up along the $c$-axis with an angle of $73^{\circ}$ on the substrate. The major driving force for the self-assembly of the $\pi$-conjugated material is the $\pi-\pi$ interaction from the adjacent molecules, which causes the superior growth direction along either the $a$-axis or $b$-axis. The measured angle of the lamellar crystal is $100.4^{\circ}$, which is consistent with the $100.2^{\circ}$ dihedral angle between the (100) and (010) planes in the crystal structure. From the crystal morphology, it can be clearly seen that the primary growth direction is along the $\pi$-stacking direction ( $b$-axis). In this direction, the slipping angle between the adjacent overlapped 2BPP units and the close contact between the adjacent $\pi$-scaffolds contributes to the significant molecular overlap and ensures the effective charge transport in the $\pi-\pi$ stacking (Fig. $3 d$ ). In addition, the intermolecular H-bond interactions are also found in the 2BPP molecular structure, which would support charge tunneling quickly from the first half to the second half. The $\pi-\pi$ stacking direction was parallel to the substrate surface according to the single crystal structure, which makes us believe that this high ordered stacking mode, with a feasible $\pi$-electron pathway, favors effective charge transport.

The crystalline ribbons grown on the substrates were fabricated as top-contact, bottom-gate configuration transistors. The
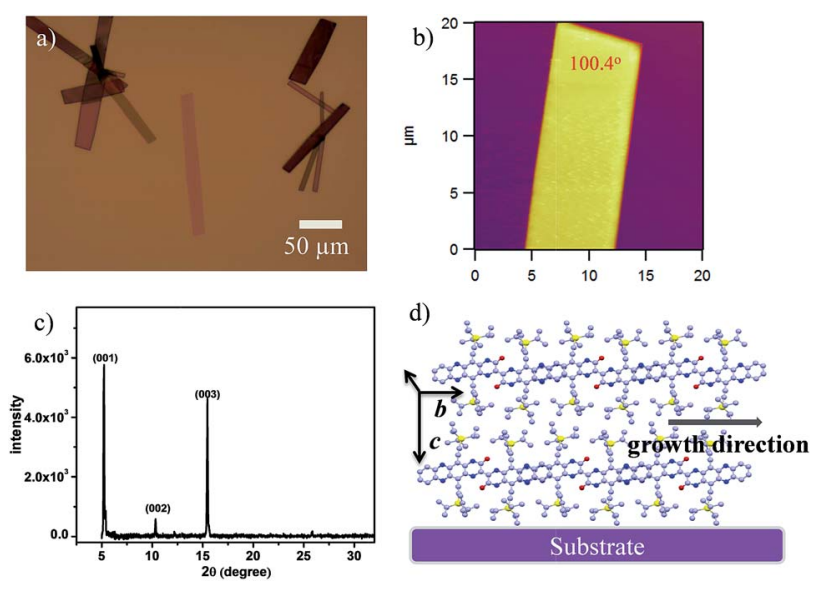

Fig. 3 (a) Optical and (b) AFM images of the self-assembled micro/nanoribbons obtained by a solution process. (c) XRD spectrum of the as-obtained micro/nanoribbons. (d) The proposed packing mode of 2BPP in the ribbons on the substrate. 
gold source and drain electrodes were deposited with copper masks covering the selected ribbons. With this simple method, $50 \mathrm{~nm}$ of Au was deposited on the covered substrate; and then, the masks were removed from the ribbon surface and the ribbon devices with a length of about $20 \mu \mathrm{m}$ were manufactured. The electrical characteristics of the devices were measured at ambient conditions. The transfer and output curves are displayed in Fig. 4a and b. The field-effect mobility $(\mu)$ was extracted from the saturation regime, and the mobility was calculated by the linear fitting of $\left(I_{\mathrm{DS}}\right)^{1 / 2}$ vs. $V_{\mathrm{G}}$ curves. Nearly 50 transistors have been measured and all the devices exhibited good gate modulation. According to the transfer characteristics, the mobility was probed in the range of $0.008-0.3 \mathrm{~cm}^{2} \mathrm{~V}^{-1} \mathrm{~s}^{-1}$ along the $b$-axis, the best hole mobility could reach $0.3 \mathrm{~cm}^{2} \mathrm{~V}^{-1} \mathrm{~s}^{-1}$ with a threshold voltage $\left(V_{\mathrm{T}}\right)$ of -10 to $-25 \mathrm{~V}$ and on-to-off current ratios $\left(I_{\text {on }} / I_{\text {off }}\right)>10^{5}$.

To understand the structure-property relationship of 2BPP, Marcus electron transfer theory and an incoherent Brownian motion model have been employed to calculate the hole and electron mobilities (see ESI $\dagger$ ) based on the single-crystal structure of 2BPP. ${ }^{11}$ The room temperature hole and electrondiffusion mobilities were predicted to be $1.49 \mathrm{~cm}^{2} \mathrm{~V}^{-1} \mathrm{~s}^{-1}$ and $1.65 \mathrm{~cm}^{2} \mathrm{~V}^{-1} \mathrm{~s}^{-1}$ (Fig. S4 and Table $\mathrm{S} 1 \dagger$ ). The simulated hole mobility is inconsistent with the measured mobility of $0.3 \mathrm{~cm}^{2} \mathrm{~V}^{-1} \mathrm{~s}^{-1}$, and the $\pi-\pi$ stacking directions show the largest transfer integrals for hole transport are $39.92 \mathrm{meV}$, which is even close to the most famous p-type semiconductor (BTBT) which has a transfer integral of $c a .60 \mathrm{meV}$. Although the material has been predicted to possess a well-balanced ambipolar property, electron transport was not apparently observed and the measured mobility was relatively low. However, we believe that with proper modification of this specific heterocyclic molecule or tuning of the device fabrication conditions, ambipolarity and good transport character could be realized.

In conclusion, we have presented the synthesis and full characterization of an unexpected "kinked" N-heteroacene (2BPP), in which two BPP units are fused together through a $\mathrm{C}=\mathrm{C}$ double bond and two $\mathrm{H}$-bonds. Single crystal X-ray studies have demonstrated that 2BPP is a near coplanar molecule with close intermolecular interactions. For the double-layer structure, the face-to-face stacking mode results in an overlap between the second layer BPP unit and the first layer unit of the second BPP molecule, forming a ladder-like corrugate. The
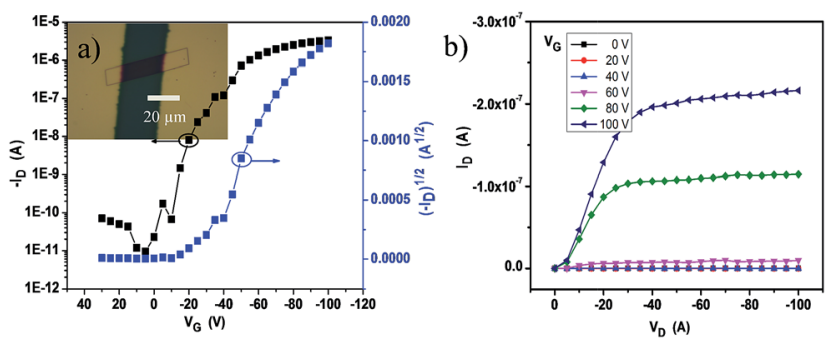

Fig. 4 (a) Typical transfer curve and (b) output curve of the single 2BPP ribbon transistors (inset, optical image of the device with an individual single crystal). electronic structure calculations suggest the unique large heterocyclic molecule could exhibit a good intrinsic ambipolar charge transport property. Experimentally, single-crystal FETs with charge carrier mobilities of $0.3 \mathrm{~cm}^{2} \mathrm{~V}^{-1} \mathrm{~s}^{-1}$ and current on/off ratios of $10^{5}$ have been realized. Further studies on the mechanism of this unusual compound as well as the hydrogen bonding supramolecular synthons would provide more insights to design and prepare novel large conjugated heteroacenes with unique properties.

\section{Acknowledgements}

The authors thank Mr Changli Chen and Prof. Zhigang Shuai from Tsinghua University for their assistance in the transfer integral calculation. The authors thank Prof. Andrew Grimsdale for his value discussion on mechanism. Q. Z. acknowledges the financial support AcRF Tier 1 (RG 133/14 and RG 13/15) and Tier 2 (ARC 2/13) from MOE, CREATE program (Nanomaterials for Energy and Water Management) from NRF, Singapore. H. Y. acknowledges the financial support by Grants-in-Aid for Scientific Research (KAKENHI) No. 25288092, 26620167 and 26105004 from the Japan Society for the Promotion of Science (JSPS).

\section{Notes and references}

1 (a) J. E. Anthony, Chem. Rev., 2006, 106, 5028-5048; (b) J. E. Anthony, Angew. Chem., Int. Ed., 2008, 47, 452-483; (c) U. H. F. Bunz, Acc. Chem. Res., 2015, 48, 1676-1686; (d) U. H. F. Bunz, J. U. Engelhart, B. D. Lindner and M. Schaffroth, Angew. Chem., Int. Ed., 2013, 52, 3810-3821; (e) J. B. Li and Q. Zhang, Synlett, 2013, 24, 686; (f) J. Li and Q. Zhang, ACS Appl. Mater. Interfaces, 2015, DOI: 10.1021/ acsami.5b00113; (g) F. Schlütter, F. Rossel, M. Kivala, V. Enkelmann, J.-P. Gisselbrecht, P. Ruffieux, R. Fasel and K. Müllen, J. Am. Chem. Soc., 2013, 135, 4550-4557; $(h)$ H. Dong, X. Fu, J. Liu, Z. Wang and W. Hu, Adv. Mater., 2013, 25, 6158-6183; (i) Y. Zhao, Y. Guo and Y. Liu, Adv. Mater., 2013, 25, 5372-5391; (j) C. Wang, J. Wang, P.-Z. Li, J. Gao, S. Y. Tan, W.-W. Xiong, B. Hu, P. S. Lee, Y. Zhao and Q. Zhang, Chem.-Asian J., 2014, 9, 779-783; (k) C. Wang, B. Hu, J. Wang, J. Gao, G. Li, W.-W. Xiong, B. Zou, M. Suzuki, N. Aratani, H. Yamada, F. Huo, P. S. Lee and Q. Zhang, Chem.-Asian J., 2015, 10, 116-119; (l) G. Giri, E. Verploegen, S. Mannsfeld, S. Atahan-Evrenk, D. H. Kim, S. Y. Lee, H. A. Becerril, A. Aspuru-Guzik, M. F. Toney and Z. Bao, Nature, 2011, 480, 504-508; $(m)$ A. L. Briseno, S. C. B. Mannsfeld, M. M. Ling, R. J. Tseng, S. H. Liu, C. Reese, M. Roberts, Y. Yang, F. Wudl and Z. Bao, Nature, 2006, 444, 913-917.

2 (a) M. Watanable, Y. J. Chang, S.-W. Liu, T.-H. Chao, K. Goto, Md. M. Islam, C.-H. Yuan, Y.-T. Tao, T. Shinmyozu and T. J. Chow, Nat. Chem., 2012, 4, 574-578; (b) J. Xiao, H. M. Duong, Y. Liu, W. Shi, L. Ji, G. Li, S. Li, X. Liu, J. Ma, F. Wudl and Q. Zhang, Angew Chem., Int. Ed., 2012, 51, 6094-6098; (c) O. D. Jurchescu, J. Baas and T. T. M. Palstra, Appl. Phys. Lett., 2004, 84, 3061-3063; (d) J. E. Anthony, 
J. S. Brooks, D. L. Eaton and S. R. Parkin, J. Am. Chem. Soc., 2001, 123, 9482-9483; (e) Q. Zhang, Y. Divayana, J. Xiao, Z. Wang, E. R. T. Tiekink, H. M. Doung, H. Zhang, F. Boey, X. Sun and F. Wudl, Chem.-Eur. J., 2010, 16, 7422-7426; $(f)$ J. Xiao, Y. Divayana, Q. Zhang, H. M. Doung, H. Zhang, F. Boey, X. Sun and F. Wudl, J. Mater. Chem., 2010, 20, 8167-8170.

3 (a) M. M. Payne, S. R. Parkin and J. E. Anthony, J. Am. Chem. Soc., 2005, 127, 8028-8029; (b) D. Chun, Y. Cheng and F. Wudl, Angew. Chem., Int. Ed., 2008, 47, 8380-8385; (c) B. Purushothaman, S. R. Parkin and J. E. Anthony, Org. Lett., 2010, 12, 2060-2063; (d) C. Tonshoff and H. F. Bettinger, Angew. Chem., Int. Ed., 2010, 49, 4125-4128; (e) B. D. Lindner, J. U. Engelhart, O. Tverskoy, A. L. Appleton, F. Rominger, A. Peters, H.-J. Himmel and U. H. F. Bunz, Angew. Chem., Int. Ed., 2011, 50, 8588-8591.

4 (a) L. Zhang, Y. Cao, N. S. Colella, Y. Liang, J.-L. Brédas, K. N. Houk and A. L. Briseno, Acc. Chem. Res., 2015, 48, 500-509; (b) L. Zhang, A. Fonari, Y. Liu, A.-L. M. Hoyt, H. Lee, D. Granger, S. Parkin, T. P. Russell, J. E. Anthony, J.-L. Brédas, V. Coropceanu and A. L. Briseno, J. Am. Chem. Soc., 2014, 136, 9248-9251.

5 (a) Q. Miao, Adv. Mater., 2014, 26(31), 5541-5549; (b) Q. Tang, D. Zhang, S. Wang, N. Ke, J. Xu, J. C. Yu and Q. Miao, Chem. Mater., 2009, 21, 1400-1405; (c) Z. Liang, Q. Tang, J. Xu and Q. Miao, Adv. Mater., 2011, 23, 1535-1539; (d) Z. Liang, Q. Tang, R. Mao, D. Liu, J. Xu and Q. Miao, Adv. Mater., 2011, 23, 5514-5518; (e) Y.-Y. Liu, C.-L. Song, W.-J. Zeng, K.-G. Zhou, Z.-F. Shi, C.-B. Ma, F. Yang, H.-L. Zhang and X. Gong, J. Am. Chem. Soc., 2010, 132, 16349-16351; (f) M. L. Tang, A. D. Reichardt, N. Miyaki, R. M. Stoltenberg and Z. Bao, J. Am. Chem. Soc., 2008, 130, 6064-6065; $(g)$ K. Takimiya, S. Shinamura, I. Osaka and E. Miyazaki, $A d v$. Mater., 2011, 23(38), 4347-4370.
6 (a) G. Li, Y. Wu, J. Gao, C. Wang, J. Li, H. Zhang, Y. Zhao, Y. Zhao and Q. Zhang, J. Am. Chem. Soc., 2012, 134, 2029820301; (b) O. Tverskoy, F. Rominger, A. Peters, H.-J. Himmel and U. H. F. Bunz, Angew. Chem., Int. Ed., 2011, 50, 3557-3560; (c) Z. He, D. Liu, R. Mao, Q. Tang and Q. Miao, Org. Lett., 2012, 14, 1050-1053; (d) C. Tong, W. Zhao, J. Luo, H. Mao, W. Chen, H. S. O. Chan and C. Chi, Org. Lett., 2012, 14, 494-497; (e) Z. He, R. Mao, D. Liu and Q. Miao, Org. Lett., 2012, 14, 4190-4193; $(f)$ P. Gu, F. Zhou, J. Gao, G. Li, C. Wang, Q. F. Xu, Q. Zhang and J. Lu, J. Am. Chem. Soc., 2013, 135, 14086-14089.

7 C. Wang, J. Zhang, G. Long, N. Aratani, H. Yamada, Y. Zhao and Q. Zhang, Angew. Chem., Int. Ed., 2015, 54, 6292-6296.

8 (a) J. Li, J. Miao, G. Long, J. Zhang, Y. Li, R. Ganguly, Y. Zhao, Y. Liu, B. Liu and Q. Zhang, J. Mater. Chem. C, 2015, 3, 1941019416; (b) J. Li, S. Chen, P. Zhang, Z. Wang, G. Long, R. Ganguly, Y. Li and Q. Zhang, Chem.-Asian J., 2015, DOI: 10.1002/asia.201500932.

9 (a) J. Strating, B. Zwanenburg, A. Wagenaar and A. C. Udding, Tetrahedron Lett., 1969, 10, 125-128; (b) M. B. Rubin and M. Kapon, J. Photochem. Photobiol., A, 1999, 124, 41-46; (c) H. Yamada, Y. Yamashita, M. Kikuchi, H. Watanabe, T. Okujima, H. Uno, T. Ogawa, K. Ohara and N. Ono, Chem.-Eur. J., 2005, 11, 6212-6220; (d) H. Yamada, Y. Yamaguchi, R. Katoh, T. Motoyama, T. Aotake, D. Kuzuhara, M. Suzuki, T. Okujima, H. Uno, N. Aratani and K.-I. Nakayama, Chem. Commun., 2013, 49, 11638-11640. 10 (a) A. Becke, J. Chem. Phys., 1993, 98, 5648-5652; (b) C. Lee, W. Yang and R. G. Parr, Phys. Rev. B: Condens. Matter Mater. Phys., 1988, 37, 785-789.

11 (a) W. Deng and W. A. Goddard, J. Phys. Chem. B, 2004, 108, 8614-8621; (b) J. L. Bredas, D. Beljonne and V. Coropceanu Cornil, Chem. Rev., 2004, 104, 4971-5004; (c) R. A. Marcus, Rev. Mod. Phys., 1993, 65, 599-610. 